\title{
Anomalous thermodynamic power laws near topological transitions in nodal superconductors
}

\author{
B. Mazidian, ${ }^{1,2}$ J. Quintanilla, ${ }^{2,3, *}$ A. D. Hillier, ${ }^{2}$ and J. F. Annett ${ }^{1}$ \\ ${ }^{1}$ H. H. Wills Physics Laboratory, University of Bristol, Tyndall Avenue, Bristol BS8 ITL, United Kingdom \\ ${ }^{2}$ ISIS facility, STFC Rutherford Appleton Laboratory, Harwell Science and Innovation Campus, Oxfordshire, OXI1 OQX, United Kingdom \\ ${ }^{3}$ SEPnet and Hubbard Theory Consortium, School of Physical Sciences, University of Kent, Canterbury CT2 7NH, United Kingdom
}

(Received 18 April 2013; revised manuscript received 18 November 2013; published 12 December 2013)

\begin{abstract}
Unconventional superconductors are most frequently identified by the observation of power-law behavior on low-temperature thermodynamic or transport properties, such as specific heat. Here, we show that, in addition to the usual point and line nodes, a much wider class of different nodal types can occur. These new types of nodes typically occur when there are transitions between different types of gap node topology, for example, when point or line nodes first appear as a function of some physical parameter. We identify anomalous, noninteger thermodynamic power laws associated with these new nodal types, and give physical examples of superconductors in which they might be observed experimentally, including the noncentrosymmetric superconductor $\mathrm{Li}_{2} \mathrm{Pd}_{3-x} \mathrm{Pt}_{x} \mathrm{~B}$.
\end{abstract}

DOI: 10.1103/PhysRevB.88.224504

PACS number(s): 74.25.Bt, 74.20.Rp, 74.25.Jb, 74.70.Tx

\section{INTRODUCTION}

A defining feature of many unconventional Fermi superfluids and superconductors is the existence of lines or points on the Fermi surface where the energy gap vanishes and so-called "nodal quasiparticles" can exist at arbitrarily low energies. In Fermi systems with such nodal quasiparticles, the low-temperature specific heat will show a particular power-law behavior as a function of temperature. The expected point and line node power-law dependencies were first derived in relation to the proposed low-temperature superfluid phases of liquid ${ }^{3} \mathrm{He} .{ }^{1}$ These were subsequently clarified further ${ }^{2}$ and are now widely used to identify pairing states in unconventional superconductors. ${ }^{3-5}$ Here, we show that gap nodes in superconductors can occur in a number of more general types than simply the usual line or point zeros and that each of these has a corresponding thermodynamic signature, typically in the form of noninteger power laws in low-temperature specific heat. We predict that these anomalous power laws generically occur at points in the phase diagram where there is a topological change in the line or point nodal structure on the Fermi surface, which we illustrate with a specific example: the noncentrosymmetric superconductors $\mathrm{Li}_{2} \mathrm{Pd}_{3-x} \mathrm{Pt}_{x} \mathrm{~B}$. In this case, the gap node topological changes are also associated with changes of bulk topological quantum numbers for the quasiparticles. The future experimental observation of such noninteger power laws could therefore be used to identify not only superconductors with highly unconventional pairing symmetries, but also topological superconducting and superfluid systems. ${ }^{6}$ Similar physics is realized in the high-temperature cobalt-doped pnictide materials $\mathrm{Ba}\left(\mathrm{Fe}_{1-x} T_{x}\right)_{2} \mathrm{As}_{2}$ $(T=\mathrm{Co}, \mathrm{Ni}, \mathrm{Pd}){ }^{7-9}$

For the usual types of point and line nodes, the quasiparticle energy spectrum is linear in the vicinity of the gap node. A familiar example is the case of the ${ }^{3} \mathrm{He}$ A phase with triplet pairing order parameter $\mathbf{d}_{\mathbf{k}} \propto\left(k_{x}+i k_{y}\right) \hat{\mathbf{z}}$, which has point nodes at the points $\mathbf{k}=\left(0,0, \pm k_{F}\right)$ on the Fermi sphere, of radius $k_{F}$. Near to these points, the quasiparticle energy spectrum, $E_{\mathbf{k}}$, obeys $E_{\mathbf{k}}^{2}=v_{F}^{2}\left(k_{z}-k_{F}\right)^{2}+\left|\mathbf{d}_{\mathbf{k}}\right|^{2}$, where $v_{F}$ is the Fermi velocity, giving a linear dispersion relation as shown in Fig. 1(a). For this type of gap node, we expect a specific heat, $C_{v} \propto T^{3}$, at temperatures $T$ much lower than the critical temperature $T_{c}$. The case of line nodes in the superconducting gap is also well known, as, for example, found in $d$-wave superconductors such as $\mathrm{YBa}_{2} \mathrm{Cu}_{3} \mathrm{O}_{7}$. Assuming in this case that the Fermi surface is an open cylinder of radius $k_{F}$, and the energy gap is of the form $\Delta_{\mathbf{k}}=\Delta_{0}\left(k_{x}^{2}-k_{y}^{2}\right)$, then there are four line nodes spaced around the Fermi surface at $\mathbf{k}=k_{F}( \pm 1, \pm 1) / \sqrt{2}$. Again, the energy spectrum is linear near these gap nodes, as shown in Fig. 1(c), so in this case we expect $C_{v} \propto T^{2}{ }^{10}$

While the generic point and line nodes of this type are expected in the majority of unconventional superconductors, other types of nodal energy spectrum are possible. For example, it is possible that the system has a point node, but that the energy spectrum is quadratic not linear near the nodal point, see Fig. 1(b). The heat capacity at this critical point is a power law, with $C_{v} \propto T^{2}$ rather than the usual $C_{v} \propto T^{3}$ for point nodes. ${ }^{2}$ This aspect of nonlinear excitation spectra was identified by Leggett in his review of the superfluid phases of ${ }^{3} \mathrm{He},{ }^{2}$ though at the time no examples were known. Later, a ground state with $E_{2 u}$ symmetry having this feature was proposed for the heavy-fermion superconductor $\mathrm{UPt}_{3}{ }^{11}$ and possible thermal transport signatures were discussed. ${ }^{12}$ Here, we show that this different type of point node behavior is not restricted to certain special symmetries but occurs generically when the gap structure is changing from having a point node to having no nodes, as a function of some parameter defining the pairing potential on the Fermi surface. We extend this notion to a much wider class of nodal types and node topology transitions.

\section{GENERALIZED NODAL TYPES AND ANOMALOUS POWER-LAWS}

The fact that it is possible to continuously remove a point node of the gap is surprising, since the usual linear spectrum point node behavior is usually thought to be topologically stable against perturbations. ${ }^{13-16}$ The shallow node behavior is not topologically protected, since it only occurs for specific parameter values in which accidental cancellations occur between different order parameter components. Nevertheless, 


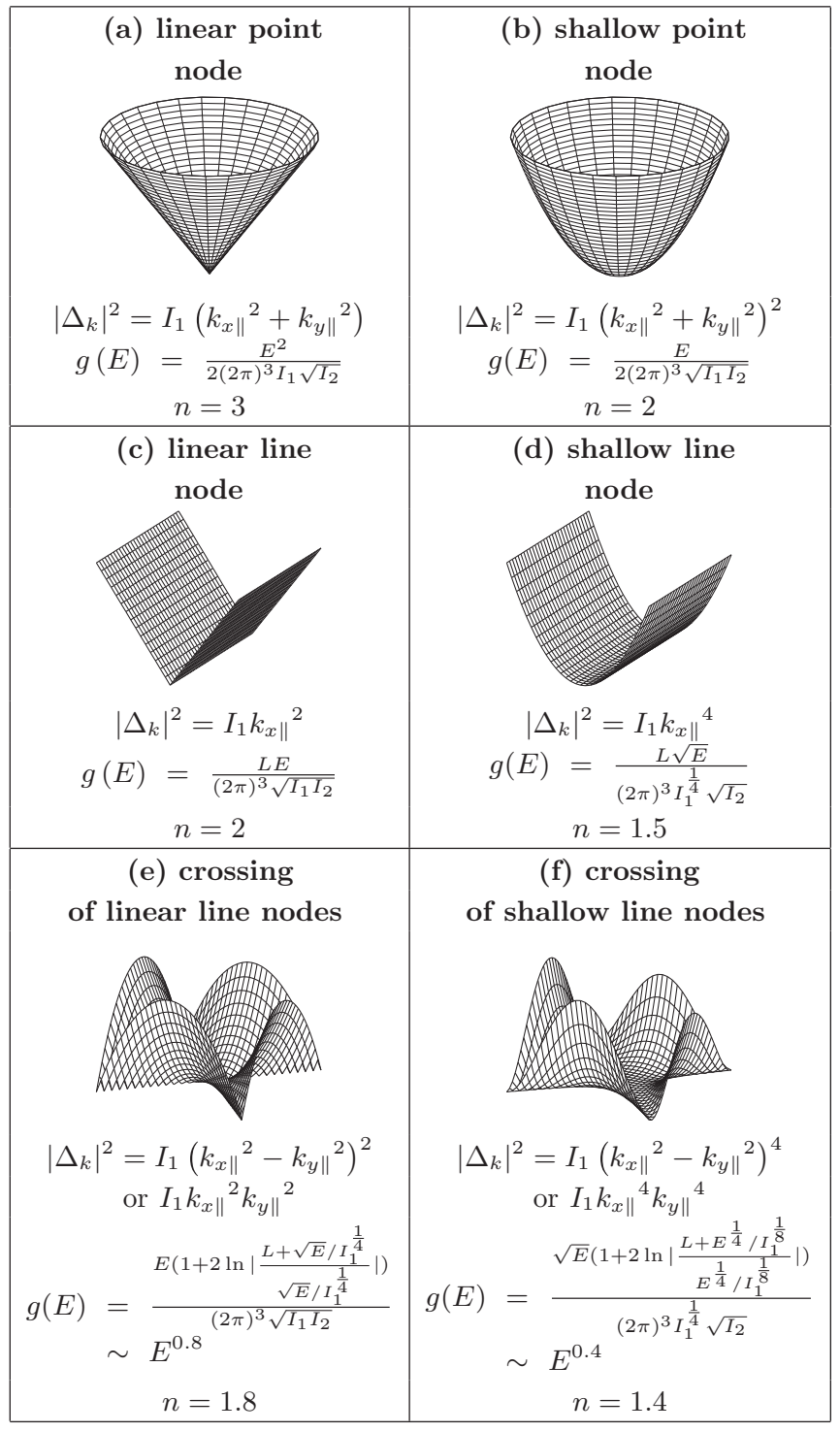

FIG. 1. The four generic types of point and line nodes in a superconductor or Fermi superfluid and the two generic types of line crossings: (a) a point node with a linear excitation spectrum, (b) a shallow point node, (c) a line node with linear spectrum, (d) a shallow line node, (e) a crossing of ordinary line nodes, and (f) a crossing of shallow line nodes. For the point and line nodes (a)-(d), we give example gap functions, densities of states and low- $T$ specific heat capacity power-law exponents $n$ for $C_{v} \propto T^{n}$. The coordinates $k_{x \|}$ and $k_{y \|}$ refer to a local coordinate system on the Fermi surface centered on the node, where $k_{z}$ is normal to the Fermi surface, and where $k_{x \|}$ and $k_{y \|}$ are coordinates tangential to the plane of the Fermi surface at the node. The crossings (e,f) each have a distinct gap spectrum, density of states and power law, which is distinct from the cases of point and line nodes alone, and which becomes dominant at low temperatures. The densities of states in these two cases are logarithmic, where the parameter $L$ denotes the length of the line nodes on the Fermi surface (measured to midway between crossing points). ${ }^{26}$ These densities of states approximate a power law, as shown, and we also show the approximate heat capacity power-law exponents $n$. Details of the derivations are given in Appendix A.

as we shall see, its influence extends throughout the finitetemperature phase diagram.
If we generalize the shallow point node behavior to line nodes, we obtain a spectrum near to the node shown in Fig. 1(d), which we will refer to as a shallow line node. In this case, we find an anomalous noninteger power law for the low-temperature specific heat, of the form $C_{v} \propto T^{1.5}$. Similarly to the shallow point node, this type of shallow line node only occurs at a boundary between a nodeless superconductor and one with ordinary line nodes, as discussed recently for the pnictide superconductors. ${ }^{7,8}$ In particular, both nodal and nodeless compounds of the system $\mathrm{Ba}\left(\mathrm{Fe}_{1-x} \mathrm{Co}_{x}\right)_{2} \mathrm{As}_{2}$ have been reported, ${ }^{17,18}$ suggesting that the $T^{1.5}$ power law could be observed experimentally. This would provide compelling evidence for the $s^{ \pm}$gap model. ${ }^{19}$

Another type of nodal behavior occurs when there is a crossing or topological reconnection of line nodes. These again lead to distinctive low-temperature power laws, with noninteger power-law exponents. A crossing of linear line nodes is illustrated in Fig. 1(e). This type of crossing can occur in a $d$-wave superconductor, for example, $\Delta_{\mathbf{k}} \propto k_{x}^{2}-k_{y}^{2}$, in the case where the Fermi surface is spherical rather than cylindrical. Although the node crossing point occurs for a small portion of the Fermi surface, it turns out that this point dominates the low-temperature heat capacity, and such crossings can therefore be detected experimentally. This is because the density of states resulting from the node crossing has a logarithmic term. This logarithmic term approximates a power law at low temperatures, and leads to a specific heat capacity contribution approximately of the form $C_{v} \propto T^{1.8}$, which at low enough temperatures dominates over the usual line node contribution $C_{v} \propto T^{2}$ arising from the line nodes away from the crossing. Unlike the shallow point node, the crossing of line nodes is expected to be topologically protected against small perturbations.

The final distinct type of gap node, which we shall discuss, is a crossing of shallow line nodes, as shown in Fig. 1(f). Again, this leads to a logarithmic term in the density of states, which dominates the low-temperature behavior compared to the shallow line node case in the absence of crossings. The corresponding specific heat capacity is approximately $C_{v} \propto$ $T^{1.4}$ for the shallow line node crossing, compared to $C_{v} \propto T^{1.5}$ for the case without crossings. Interestingly, $n=1.4$ is not so far from the value obtained for the ungapped Fermi surface of a normal (nonsuperconducting) Fermi liquid, $n=1$.

Figure 2 shows the predicted exponents of the power-law temperature dependencies of specific heat for each of the different nodal types shown in Fig. 1. In all cases, the predicted power-law behavior $C_{v} \propto T^{n}$ is accurate at very low temperatures, below $0.1 T_{c}$, and in many cases the lowtemperature power laws apply over a range of temperatures up to $0.3 T_{c}$. The details of our calculations of specific heat leading onto the results in Figs. 1 and 2 are given in Appendix A.

Non-integer power laws, especially powers below 2 , are a highly distinctive signature of the low-energy excitation spectrum, and their experimental observation would be a relatively clear and direct form of evidence for the existence of the corresponding type of gap node in a given material. In systems where the gap topology is changing as a function of some experimental parameter (e.g., doping or spin-orbit coupling), these anomalous powers will only be observed to 


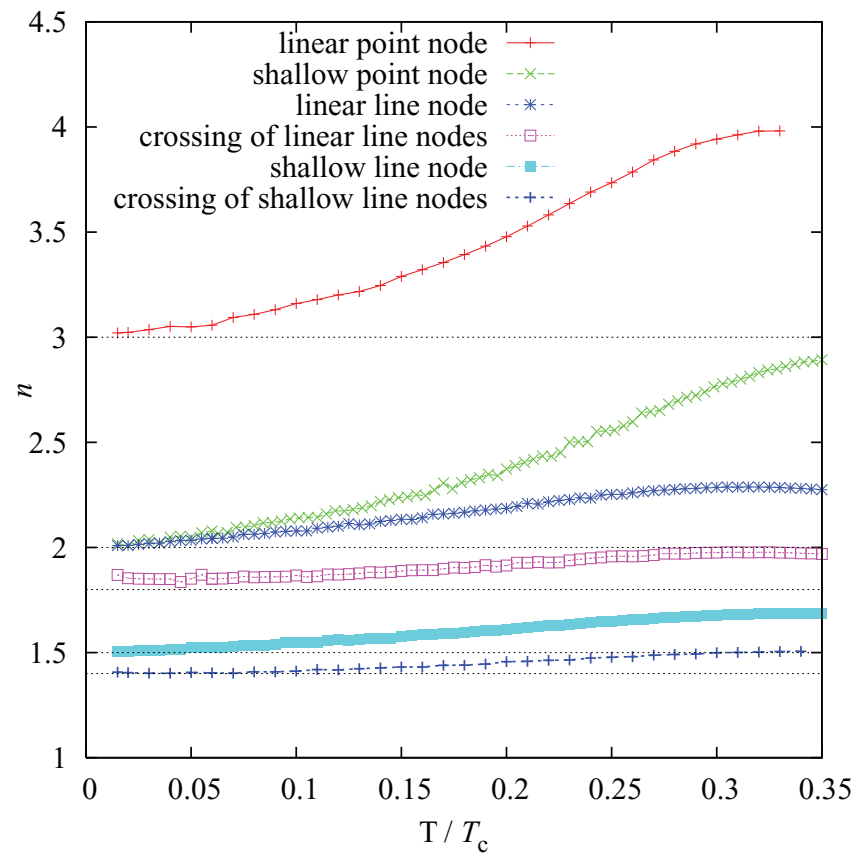

FIG. 2. (Color online) The simulated exponent of temperature $T$ in the specific heat capacity as a function of temperature for each of the nodal characters. The value of $n$ in the power law $C_{v} \propto T^{n}$ is obtained by calculating $n=\frac{d \ln \left(C_{v}\right)}{d \ln (T)}$. These match analytics (dotted lines) in the limit $T \rightarrow 0$. The methodology employed to produce this plot is described in Appendix A.

the lowest temperatures if the material is exactly at the critical parameter value for the shallow node to occur. However, if the material is merely near to, not exactly at, the critical parameter value, then the shallow node power laws will hold over a wide temperature range, reverting to ordinary line, point, or nodeless behavior at the very lowest temperatures. Again, experimental observation of such a crossover between power laws would be a clear signature of the unusual gap nodal structure-we provide an explicit example of this below.

In practice, it is unlikely that the power laws shown in Fig. 2 can be distinguished from one another in a single measurement as a function of temperature, however, a transition of the type shown in Fig. 5 (inset), discussed below, is more feasible as a function of doping at constant temperature.

\section{ANOMALOUS POWER-LAWS THROUGH NODAL RECONFIGURATION TRANSITIONS}

In addition to the ad hoc mechanisms mentioned above, we expect shallow line or point nodes to occur generically in superconductors with multicomponent order parameters. Consider the simple model shown in Fig. 3. This shows a hypothetical system, such as an ordinary $s$-wave BCS superconductor with a significant level of anisotropy in the gap. Here, $\Delta_{0}$ is the average gap and $\Delta_{1}$ is a measure of the gap variation with $k_{\|}$parallel to the Fermi surface. In the case where $\left|\Delta_{0}\right|>\left|\Delta_{1}\right|$, the gap is nodeless everywhere on the Fermi surface. On the other hand, if $\left|\Delta_{0}\right|<\left|\Delta_{1}\right|$ then the gap changes sign at some points on the Fermi surface, leading to pairs of line nodes. At the critical parameter value $\left|\Delta_{0}\right|=\left|\Delta_{1}\right|$, the line node pairs coalesce, leading to a single, shallow, line node. In this example, no symmetry change occurs in the gap function, however, "accidental" line nodes occur within a single symmetry of the Cooper pair order parameter because of the large degree of gap anisotropy.

An explicit example of this mechanism by which anomalous power laws may be observed is provided by noncentrosymmetric superconductors where the superconductor order parameter

$$
\hat{\Delta}_{\mathbf{k}}=\left(\Delta_{0, \mathbf{k}}+\mathbf{d}_{\mathbf{k}} \cdot \boldsymbol{\sigma}\right) i \hat{\sigma}_{y}
$$

has singlet and triplet components $\Delta_{0, \mathbf{k}}$ and $\mathbf{d}_{\mathbf{k}}$, respectively, such as the compounds $\mathrm{Li}_{2} \mathrm{Pd}_{3-x} \mathrm{Pt}_{x} \mathrm{~B} \cdot{ }^{20-22}$ Platinum doping in this system is thought to increase the spin-orbit coupling, and hence increase the triplet component of the order parameter relative to the singlet component. ${ }^{22-25}$ In doing so, the spin-orbit coupling introduces nodes in the gap. Within a standard symmetry classification of the possible order parameter structures, the simplest one takes the form ${ }^{27,28}$

$$
\begin{gathered}
\Delta_{0, \mathbf{k}}=\Delta_{0} \\
\mathbf{d}_{\mathbf{k}}=\Delta_{0}\{A[X, Y, Z] \\
\left.-B\left[X\left(Y^{2}+Z^{2}\right), Y\left(X^{2}+Z^{2}\right), Z\left(Y^{2}+X^{2}\right)\right]\right\},
\end{gathered}
$$

where $X, Y, Z$ are functions with the same symmetry properties as the components of the electron wave vector $k_{x}, k_{y}, k_{z}$ and $A$ and $B$ are material-specific parameters that depend on doping $x$. Equations (2) and (3) correspond to the $A_{1}$ irreducible representation of the cubic $O$ group, which is the only one where only gauge symmetry is broken. Other pairing states break additional symmetries and therefore are not compatible
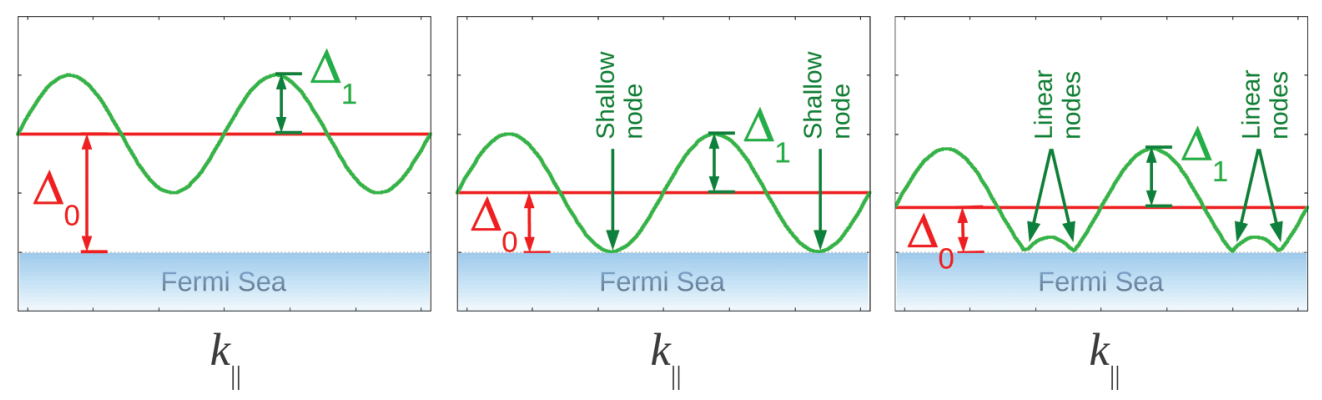

FIG. 3. (Color online) The transition between a nodal and nonnodal gap structure in any superconductor as a result of continuously admixed order parameters will be characterized by a nonlinear gap energy spectrum close to the node. This is because in any wavelike order parameter the deep minima have no linear order term in the expansion. 

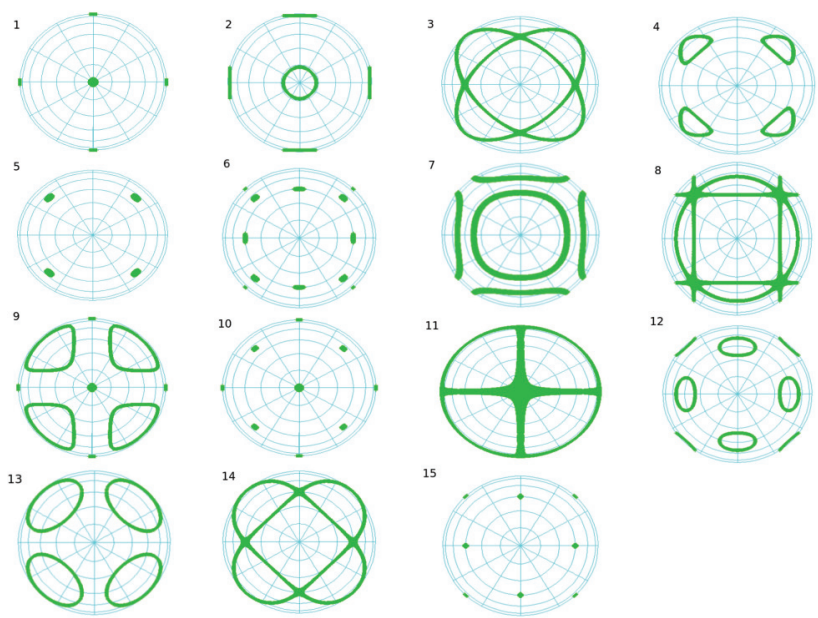

15
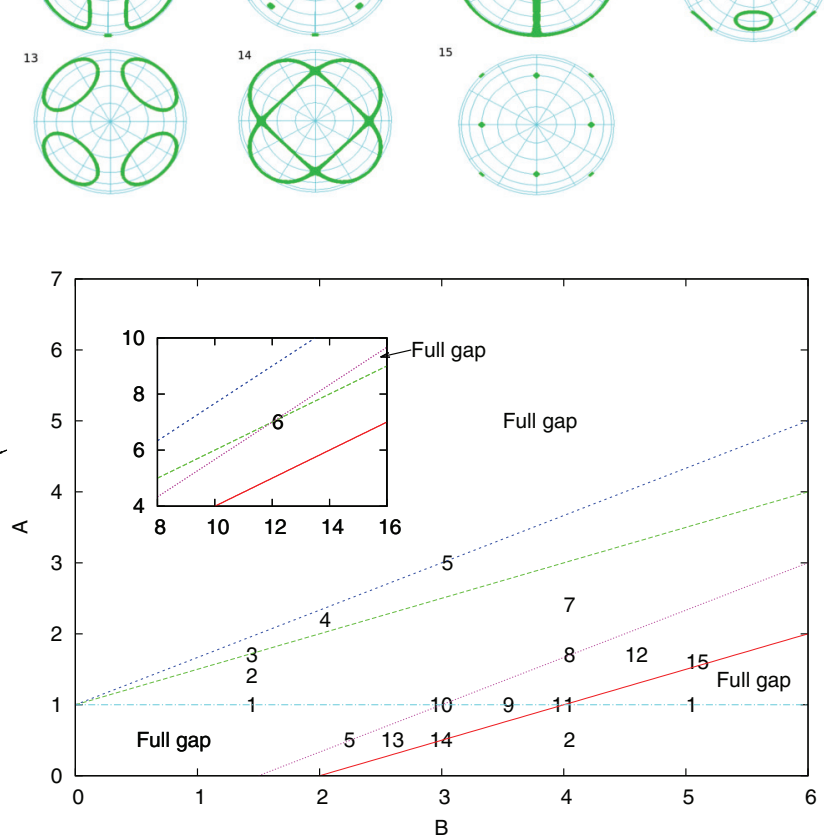

FIG. 4. (Color online) (Top) Fifteen gap topologies corresponding to different triplet admixtures in a cubic noncentrosymmetric superconductor. Within this set, the only point nodes are shallow point nodes, occurring in plots $1,5,10$, and 15 . Topologies $3,6,8$, and 14 are all line reconnection transitions between distinct topologies and include crossings of linear line nodes. Topology 11 is a crossing of shallow line nodes. (Bottom) Phase diagram of gap node topology corresponding to triplet admixture parameters $A$ and $B$ in Eq. (3) on a spherical Fermi surface. Each line represents a phase boundary where gap nodes appear or disappear on the Fermi surface.

with a conventional (phonon-mediated) pairing mechanism. In spite of this, a large number of different gap nodal structures are obtained, as shown in Fig. 4 (top panel). As a function of the parameters $A, B$ we obtain the rich phase diagram of Fig. 4 (bottom panel). The corresponding specific heat exponents are illustrated in Fig. 5. Upon doping, the material explores the phase diagram along some unspecified path from small values of $A$ and $B(x=0)$ to larger values $(x=3) .{ }^{22}$ Small values of $A$ and $B$ correspond to a fully gapped, singlet-dominated superconducting order parameter as believed to be realized in $\mathrm{Li}_{2} \mathrm{Pd}_{3} \mathrm{~B}$. Order parameters with a large triplet component and line nodes on the Fermi surface are thought to occur in $\mathrm{Li}_{2} \mathrm{Pt}_{3} \mathrm{~B}$ and correspond to the case when either $A$ or $B$ (or both) are larger.

Inspection of the phase diagram shows that at some values of $A$ and $B$ we expect point nodes to appear, labeled by
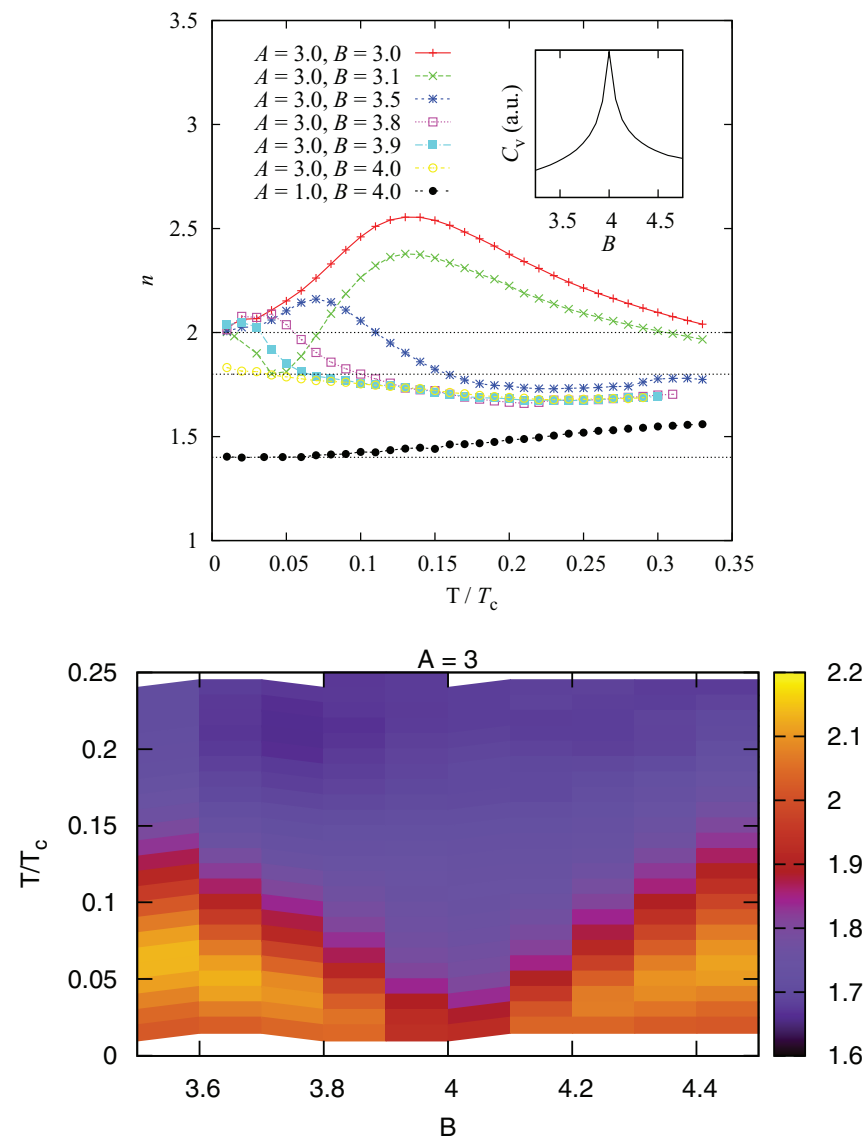

FIG. 5. (Color online) (Top) Specific heat exponent as a function of temperature for different values of the $A$ and $B$ parameters, as indicated. The inset shows the value of the specific heat as a function of $B$ for fixed $A=3$ and constant temperature, $T=0.005 T_{c}$. (Bottom) Specific heat exponent as a function of temperature and parameter $B$ for fixed $A=3$. Details of the calculation of the quasiparticle spectrum and phase diagram are given in Appendix A.

"1" in Fig. 4, before line nodes appear on the gap, labeled by " 2 " in the same figure. These point nodes are shallow point nodes, and so instead of the usual $T^{3}$ power-law dependence of the heat capacity for point nodes, we instead expect a $T^{2}$ power-law dependence. Upon further change of the $A$ and $B$ parameters, these shallow point nodes expand into rings of ordinary line nodes, as shown in Fig. 4, plot labeled " 2 ." Increasing the parameters yet again there is a level which causes a topological line reconnection, labeled " 3 ," where the different line nodes intersect before becoming rings again, now centered at different points on the Fermi surface, "4." Note that the number of line nodes on the Fermi surface is always a multiple of two as predicted by the topological theory of gapless phases in time-reversal-invariant superconductors. ${ }^{29}$ That number jumps at the line reconnection transition, indicating its topologically nontrivial nature. The line reconnection transition has a distinct thermal signature in the low- $T$ heat capacity originating from the crossing of line nodes associated with such a line reconnection. As is evident from the figure, there are many other paths along the phase diagram with similar phase transitions. In our analysis of the cubic noncentrosymmetric superconductors, we also found a 
single instance of crossing of shallow line nodes, labeled " 11 " in Fig. 4. These are realized when line nodes are spontaneously introduced on a nodeless Fermi surface without first appearing as points. The specific heat exponent of $n=1.4$ for this case is clearly seen in Fig. 5.

Schnyder et al. ${ }^{27,28}$ have shown that a number of these phases of cubic noncentrosymmetric superconductors possess nontrivial topological quantum numbers, ${ }^{6,29}$ and have corresponding surface edge states of topological nature. In principle, these surface states can be probed experimentally by electron tunneling through an interface with a normal metal. ${ }^{30}$ However, we propose that a more direct, bulk, measurement of this nontrivial gap topology can be obtained by measurements of the low-temperature heat capacity. If the anomalous power laws we have predicted can be observed, then it should be possible to detect the phase boundaries between different types of topological gap structures, and hence determine in a bulk measurement the topological quantum numbers associated with different doping regimes. Phases $1-5$ in Fig. 4 correspond to those considered by Schnyder et al. ${ }^{27,28}$

The anomalous power-laws due to changes of nodal topology are realized exactly at each transition. On the other hand, by being close to, but not exactly at one of those transitions one expects a crossover from conventional (fully gapped or normal power-law) behavior to anomalous behavior as the temperature is increased through a characteristic temperature scale $T^{*}$. A demonstration of this is provided for the case of $\mathrm{Li}_{2} \mathrm{Pd}_{3-x} \mathrm{Pt}_{x} \mathrm{~B}$, see Fig. 5, specifically the curves corresponding to $A=3$, $B=3.5-4.0$ (top panel), and the density plot (bottom panel) showing how the temperature scale $T^{*}$ converges to zero at the transition. This may be used to detect topological transitions in superconductors and in fact shows that the influence of the topological transition extends over a wide region in the phase diagram, not just at a single point. The detection of the phase boundary itself is facilitated by its higher specific heat, at fixed low temperature, due to the enhanced phase space available to quasiparticles in that state. This is illustrated in the inset to Fig. 5. We emphasize, however, that it is in the anomalous power laws that we find the evidence of the special nature of quasiparticles at the phase boundary.

In recent years, it has become widely recognized that point or line nodes in the bulk spectrum of topological matter may evolve into topologically protected surface or vortex core states with zero dispersion. ${ }^{31,32}$ In contrast, the shallow dispersions discussed here exist in the bulk excitation spectrum near topological transitions.

\section{CONCLUSION}

To conclude, we have shown that a more general classification of nodal characters of superconductors yields anomalous, noninteger power laws for low-temperature thermodynamic quantities. Some such power laws can be used to detect line node crossings; others are expected at phase transitions where the nodal topology reconfigures itself. An example is provided by the topological transitions expected to occur as a function of doping in the noncentrosymmetric cubic superconductor $\mathrm{Li}_{2} \mathrm{Pd}_{3-x} \mathrm{Pt}_{x} \mathrm{~B}$. It is important to stress that the observation of anomalous low-temperature power laws, just as the ordinary, integer ones, requires going to temperatures well below $T_{c}$. On the other hand, it is not necessary to sit exactly at the transition, as there is a crossover temperature separating conventional from anomalous power-law behavior that converges to zero there. In that sense, the influence of the topological transition extends throughout the bulk phase diagram in a manner similar to that of a quantum critical endpoint. ${ }^{33,34}$

\section{ACKNOWLEDGMENTS}

This works was supported by EPSRC and STFC (UK). J.Q. gratefully acknowledges funding from HEFCE and STFC through the South-East Physics network (SEPnet).

\section{APPENDIX A: CALCULATION OF SPECIFIC HEAT OF NODAL SUPERCONDUCTORS}

To describe the energy $E_{\mathbf{k}}$ of quasiparticles near any specific point on the Fermi surface, it is convenient to change coordinate systems to three mutually orthogonal kvector components: one perpendicular to the Fermi surface, $k_{\perp}$, and two parallel to it, $k_{x \|}, k_{y \|}$. We then have $E_{\mathbf{k}}^{2}=$ $v_{F}^{2} k_{\perp}^{2}+\Delta_{\mathbf{k}}^{2}$, where $v_{F}$ is the Fermi velocity, $k_{\perp}=0$ when $|\mathbf{k}|=k_{F}$ (the Fermi vector), and $\Delta_{\mathbf{k}}$ is a function determined by the superconducting gap function, which may be either singlet, triplet, or a combination (for noncentrosymmetric systems).

The gap energy spectra shown in Fig. 1 are described by an equation for the gap energy $\Delta_{\mathbf{k}}$, which has the form $\Delta_{\mathbf{k}}^{2}=$ $I_{\mu \nu} k_{\mu \|} k_{\nu \|}+\cdots$ near to a gap nodal point, where $I_{\mu \nu}$ is a positive tensor of rank 2 , which is determined by the symmetry of the gap function around that nodal point. In general, $I_{\mu \nu}$ will have two principal axes, which we can choose as $k_{x \|}$ and $k_{y \|}$. If the two eigenvalues corresponding to these axes, $I_{x}$ and $I_{y}$ are both positive and nonzero, we have an ordinary point node with linear spectrum illustrated in Fig. 1(a) (with $I_{1}=\sqrt{I_{x} I_{y}}$ ). If one eigenvalue is positive and nonzero $\left(I_{1}\right)$ and the other is zero we have an ordinary line node. In the case of two zero eigenvalues, it is necessary to continue the expansion to quartic or higher order, giving the various shallow nodes or crossings illustrated in Fig. 1. In principle, even higher powers may be possible, but we have not found any realistic examples of gap models where this could occur. In contrast, all the node types presented in Fig. 1 can be realized in the specific gap models presented in this paper.

Computation of the density of states integral $g(E)=$ $\iiint \delta\left(E_{\mathbf{k}}-E\right) d k_{x} d k_{y} d k_{z}$ for each of these gap energy spectra gives a unique expression. The specific heat capacity in the superconducting state is in turn given by ${ }^{2}$

$$
\begin{aligned}
C_{v} & \equiv T\left(\frac{d S}{d T}\right) \\
& =\sum_{\mathbf{k}} \frac{1}{2} k_{B} \beta^{2}\left[E_{\mathbf{k}}+\beta\left(\frac{d E_{\mathbf{k}}}{d \beta}\right)\right] E_{\mathbf{k}} \operatorname{sech}^{2}\left(\frac{\beta E_{\mathbf{k}}}{2}\right),
\end{aligned}
$$

where $\beta=1 / k_{B} T$. The low- $T$ specific heat capacity can therefore be found analytically from $g(E)$ by evaluating the integral $\frac{1}{2} k_{B} \beta^{2} \int_{0}^{\infty} d E g(E) E^{2} \operatorname{sech}^{2}(\beta E / 2)$, giving the power-law dependence of a low- $T$ specific heat capacity 
measurement. We find the terms listed in Fig. 1 for point and line nodes with linear and nonlinear excitation spectra, where $L$ is the length of the line node from end to end. For cases where line nodes cross, we expect the terms listed in Fig. 1, where $L$ is now the length of the line node on the Fermi surface measured from the center to the corner of the crossing whose contribution is being calculated. The cases in Figs. 1(e) and 1(f) are not power laws, however, they approximate very closely to the noninteger power laws indicated, with very slight variations depending on the constant $L$. In the case of line reconnection transitions such as Fig. 4, plots 3, 8, and 14, where the Fermi surface includes regions where the gap energy spectrum expands as $\Delta_{\mathbf{k}}^{2}=I_{1} k_{x}{ }^{2} k_{y \|}{ }^{2}$, the contribution of the line crossing to the density of states is $\sim E^{0.8}$.

The low- $T$ specific heat capacity can also be calculated numerically using Eq. (A1) by summing over a sufficient number of integration points corresponding to different values of the gap in $k$ space near the Fermi surface for selected temperature ranges below $\mathrm{T}_{c}$. The exponent of temperature, $n$, can then be obtained using the general formula $n=$ $d \ln C_{v} / d \ln T$. When this quantity approaches a constant, we have $C_{v} \propto T^{n}$. This yields the curves in Figs. 2 and 4(c), where the temperature dependence of the gap is taken to be given by the expression from Ginzburg-Landau theory, $\left(T-T_{c}\right)^{1 / 2}$. This approximation, made for convenience, introduces a weak additional temperature dependence for $T \ll T_{c}$ compared to the BCS approximation and therefore provides an upper bound on the temperature dependence introduced by the gap. We find that the exponents are not affected by this: even in the case where the temperature dependence of the gap is assumed to be constant, the same power laws are obtained with flat temperature dependence.

Each contour in Fig. 4(a) corresponds to a line where the gap energy $\Delta_{\mathbf{k}}$ derived from the gap function of Eqs. (2) and (3) vanishes. This gap energy is given by $\left|\Delta_{\mathbf{k}}\right|=\left|\Delta_{0} \pm d(\mathbf{k})\right|=0$, where $d(\mathbf{k})=\left|\mathbf{d}_{\mathbf{k}}\right|$. The phase diagram in Fig. 4(b) shows the values of the parameters $A$ and $B$ where the nodal topology changes for this form of the gap energy. This form of $\Delta_{\mathbf{k}}$ has also been used to compute the specific heat exponent in Fig. 4(c).

\section{APPENDIX B: SPECTRUM OF $O$-GROUP SUPERCONDUCTORS}

Here, we give the details of the derivation of the nodal topologies and phase diagram presented in Fig. 4(b). We use a single-band mean-field model with antisymmetric spin-orbit coupling to obtain the resulting gap node topologies on the Fermi surface, which we take to be spherical for simplicity. The Bogoliubov-de Gennes Hamiltionian is

$$
H(\mathbf{k})=\left(\begin{array}{cc}
\hat{h}(\mathbf{k}) & \hat{\Delta}(\mathbf{k}) \\
\hat{\Delta}^{\dagger}(\mathbf{k}) & -\hat{h}^{T}(-\mathbf{k})
\end{array}\right) .
$$

The noncentrosymmetric (NCS) gap function, which mixes singlet and triplet as a result of the parity violation in the crystal lattice structure, is given in Eq. (1), where $\sigma_{x, y, z}$ denotes the Pauli matrices and $\Delta_{0, \mathbf{k}}$ and $\mathbf{d}_{\mathbf{k}}$ denote the singlet and triplet components of the gap, respectively.
The normal state Hamiltonian $h(\mathbf{k})$ given in Ref. 27 for noninteracting electrons in a crystal without inversion center is

$$
h(\mathbf{k})=\varepsilon_{\mathbf{k}} I+\gamma_{\mathbf{k}} \cdot \sigma,
$$

where $\varepsilon_{\mathbf{k}}=\varepsilon_{-\mathbf{k}}$ is the nonrelativistic metallic dispersion energy. The second term represents the antisymmetric SOC where the coupling constant $\boldsymbol{\gamma}_{\mathbf{k}}=-\boldsymbol{\gamma}_{-\mathbf{k}}$. We will assume the $d$-vector $\mathbf{d}_{\mathbf{k}}$ to be parallel to $\boldsymbol{\gamma}_{\mathbf{k}}$. This ensures that SOC is not destructive to the triplet component of the NCS gap function. ${ }^{27,35}$

In a general Ginzburg-Landau theory, the possible symmetries of the superconducting instability depend on the symmetry of the normal state. In this case, the symmetry of the normal state is given by $G_{N}=O \times T \times U(1)$ where $O$ denotes the NCS cubic space group, $T$ the time reversal symmetry and $U(1)$ the gauge symmetry operation. In any superconducting transition, the $U(1)$ symmetry is broken, however, other symmetries of the normal state may be broken as well. For the purposes of our analysis, we will, following Ref. 27 , look at the case where only the gauge symmetry and no further symmetries of the crystal lattice are broken, i.e., the superconducting instability has the symmetry of the $A_{1}$ irrep of the $O$ group. This means that the gap function, given, in general, by Eq. (1), takes the specific form of Eqs. (2) and (3).

With $\mathbf{d}_{\mathbf{k}}$ and $\boldsymbol{\gamma}_{\mathbf{k}}$ explicitly defined, the Bogoliubov deGennes (BdG) Hamiltonian can be diagonalized. The singleband BdG Hamiltonian with SOC, defined parallel to the $\mathbf{d}(\mathbf{k})$ component of the gap, ${ }^{27,35}$ has the property that splitting of the Fermi surface occurs as a result of SOC, whereas zero-energy excitations occur as a result of the combination of singlet and triplet components of the gap function. In this case, the diagonalized $\mathrm{BdG}$ Hamiltonian has eigenvalues of the form

$$
E= \pm \sqrt{\left(\varepsilon_{\mathbf{k}}-\mu \pm\left|\boldsymbol{\gamma}_{\mathbf{k}}\right|\right)^{2}+\left|\Delta_{0} \pm\right| \mathbf{d}_{\mathbf{k}}||^{2}}
$$

where the \pm signs inside the square root always take the same value, while the sign in the front varies independently. The SOC term $\boldsymbol{\gamma}_{\mathbf{k}}$ in this expression has the effect of splitting Fermi sheets, whereas the second term $\left|\Delta_{0} \pm\right| \boldsymbol{d}_{\mathbf{k}}||$ determines the node topology of the superconducting gap on either of the Rashba-split Fermi sheets.

The zeros of the term $\left|\Delta_{0} \pm\right| d_{\mathbf{k}}||$ are the loci of zeroenergy excitations on the Fermi surface. In order to ensure that the SOC parameter does not significantly warp the spherical Fermi surface and therefore change the node topology, we assume that $\epsilon_{\mathbf{k}} \gg \boldsymbol{\gamma}_{\mathbf{k}} \gg \Delta_{0}$. This hierarchy of energy scales is reasonable to assume as $\epsilon_{\mathbf{k}}$ is of the order of $\mathrm{eV}, \Delta_{0}$ is of the order of meV, and the observed SOC-induced Fermi surface splittings are small relativistic corrections to $\epsilon_{\mathbf{k}}$.

The conditions for zeros in the gap energy spectrum for each Rashba-split Fermi sheet are $\Delta_{0}-\left|\mathbf{d}_{\mathbf{k}}\right|=0$ and $\Delta_{0}+\left|\mathbf{d}_{\mathbf{k}}\right|=$ 0 . Thus, one of the Fermi surface sheets is always fully-gapped, while the other one may be nodal. The gap function in this latter case is best normalized with respect to the singlet gap energy:

$$
\Delta_{0}^{2}\left|1-\frac{\left|\mathbf{d}_{\mathbf{k}}\right|}{\Delta_{0}}\right|^{2}=\Delta_{0}^{2}|1-| \mathbf{d}_{\mathbf{k}}^{\prime}||^{2}=0 .
$$


The term $\frac{\left|\mathbf{d}_{\mathbf{k}}\right|}{\Delta_{0}}$ has here been replaced with the term $\left|\mathbf{d}_{\mathbf{k}}^{\prime}\right|$ whose coefficients are scaled by a factor of $\Delta_{0}$. This expression can be fully expanded and used to derive the topological phase boundaries by substituting in the vector $\mathbf{d}^{\prime}(k)=[A X-$ $\left.B X\left(Y^{2}+Z^{2}\right), A Y-B Y\left(Z^{2}+X^{2}\right), A Z-B Z\left(X^{2}+Y^{2}\right)\right]$. A coordinate transformation to spherical polar coordinates using $X=\cos (\phi) \sin (\theta), Y=\sin (\phi) \sin (\theta)$, and $Z=\cos (\theta)$ allows us to select specific points on the Fermi surface and find solutions in A and B. The phase boundaries of Fig. 4(b) separate regions of constant gap topology, which were obtained for critical values of the polar and azimuthal angles corresponding to the points where nodes first appear on the gap or where line nodes reconnect at a topological transition.
Phases 1-5 in Fig. 4(a) of the main text correspond to those considered by Schnyder et al. ${ }^{27,28}$ The coordinates $\theta=\cos ^{-1}(1 / \sqrt{3}), \phi=\frac{\pi}{4}$ correspond to one topologically distinct set of point nodes on the spherical Fermi surface shown in Fig. 4(a), plot 5. Substituting these into the equation $\Delta_{0}-$ $\left|\mathbf{d}_{\mathbf{k}}\right|=0$ gives the relationship between coefficients $A$ and $B$ : $A=\frac{2}{3} B \pm 1$. The line reconnection shown in Fig. 4(a), plot 3, corresponds to the coordinates $\theta=\frac{\pi}{4}, \phi=0$ and produces solutions $A=\frac{B \pm 2}{2}$. The final phase boundary in Fig. 4(b) corresponds to point nodes at the coordinates $\theta=\frac{\pi}{2}, \phi=0$ shown in Fig. 4(a), plot 1, which produces the solutions $A= \pm 1$. *j.quintanilla@kent.ac.uk

${ }^{1}$ P. W. Anderson and P. Morel, Phys. Rev. 123, 1911 (1961).

${ }^{2}$ A. J. Leggett, Rev. Mod. Phys. 47, 331 (1975).

${ }^{3}$ J. F. Annett, Adv. Phys. 39, 83 (1990).

${ }^{4}$ M. Sigrist and K. Ueda, Rev. Mod. Phys. 63, 239 (1991).

${ }^{5}$ A. P. Mackenzie and Y. Maeno, Rev. Mod. Phys. 75, 657 (2003).

${ }^{6}$ A. P. Schnyder, S. Ryu, A. Furusaki, and A. W. W. Ludwig, Phys. Rev. B 78, 195125 (2008).

${ }^{7}$ Rafael M. Fernandes and Jörg Schmalian, Phys. Rev. B 84, 012505 (2011).

${ }^{8}$ Valentin Stanev, Boian S. Alexandrov, Predrag Nikolic, and Zlatko Tesanovic, Phys. Rev. B 84, 014505 (2011).

${ }^{9}$ V. Mishra, G. Boyd, S. Graser, T. Maier, P. J. Hirschfeld, and D. J. Scalapino, Phys. Rev. B 79, 094512 (2009).

${ }^{10}$ N. E. Hussey, Adv. Phys. 51, 1685 (2002).

${ }^{11}$ J. A. Sauls, Adv. Phys. 43, 113 (1994).

${ }^{12}$ M. J. Graf, S. K. Yip, J. A. Sauls, and D. Rainer, Phys. Rev. B 53, 15147 (1996); Yu. S. Barash and A. A. Svidzinsky, ibid. 58, 6476 (1998); V. P. Mineev K. V. Samokhin, Introduction to Unconventional Superconductivity (Gordon and Breach Science Publishers, Amsterdam, 1999); H. Shakeripour, C. Petrovic, and Louis Taillefer, New J. Phys. 11, 055065 (2009).

${ }^{13}$ G. E. Volovik, The Universe in a Helium Droplet (Oxford University Press, Oxford, 2003).

${ }^{14}$ G. E. Volovik, Pis'ma Zh. Eksp. Teor. Fiz. 46, 81 (1987) [JETP Lett. 46, 98 (1987)].

${ }^{15}$ M. Sato, Phys. Rev. B 73, 214502 (2006).

${ }^{16}$ G. E. Volovik, Lect. Notes Phys. 718, 31 (2007).

${ }^{17}$ K. Gofryk, A. S. Sefat, M. A. McGuire, B. C. Sales, D. Mandrus, J. D. Thompson, E. D. Bauer, and F. Ronning, Phys. Rev. B 81, 184518 (2010).
${ }^{18}$ M. A. Tanatar, J.-P. Reid, H. Shakeripour, X. G. Luo, N. Doiron-Leyraud, N. Ni, S. L. Bud'ko, P. C. Canfield, R. Prozorov, and L. Taillefer, Phys. Rev. Lett. 104, 067002 (2010).

${ }^{19}$ P. J. Hirschfeld, M. M. Korshunov, and I. I. Mazin, Rep. Prog. Phys. 74, 124508 (2011).

${ }^{20}$ K. Togano, P. Badica, Y. Nakamori, S. Orimo, H. Takeya, and K. Hirata, Phys. Rev. Lett. 93, 247004 (2004).

${ }^{21} \mathrm{P}$. Badica, T. Kondo, T. Kudo, Y. Nakamori, S. Orimo, and K. Togano, Appl. Phys. Lett. 85, 4433 (2004).

${ }^{22}$ H. Q. Yuan, D. F. Agterberg, N. Hayashi, P. Badica, D. Vandervelde, K. Togano, M. Sigrist, and M. B. Salamon, Phys. Rev. Lett. 97, 017006 (2006).

${ }^{23}$ M. Nishiyama, Y. Inada, and Guo-qing Zheng, Phys. Rev. Lett. 98, 047002 (2007).

${ }^{24}$ H. Takeya, M. ElMassalami, S. Kasahara, and K. Hirata, Phys. Rev. B 76, 104506 (2007).

${ }^{25}$ S. Harada, J. J. Zhou, Y. G. Yao, Y. Inada, and Guo-qing Zheng, Phys. Rev. B 86, 220502(R) (2012).

${ }^{26}$ B. Mazidian, Ph.D. thesis, University of Bristol, 2013.

${ }^{27}$ A. P. Schnyder and S. Ryu, Phys. Rev. B 84, 060504(R) (2011).

${ }^{28}$ A. P. Schnyder, P. M. R. Brydon, and C. Timm, Phys. Rev. B 85, 024522 (2012).

${ }^{29}$ B. Béri, Phys. Rev. B 81, 134515 (2010).

${ }^{30}$ P. M. R. Brydon, A. P. Schnyder, and C. Timm, Phys. Rev. B 84, 020501(R) (2011).

${ }^{31}$ T. T. Heikkila, N. B. Kopnin, and G. E. Volovik, Pis'ma Zh. Eksp. Teor. Fiz. 94, 252 (2011).

${ }^{32}$ T. T. Heikkila and G. E. Volovik, JETP Lett. 93, 59 (2011).

${ }^{33}$ S. A. Grigera et al., Science 294, 329 (2001).

${ }^{34}$ A. Hackl and M. Vojta, Phys. Rev. Lett. 106, 137002 (2011).

${ }^{35}$ P. A. Frigeri, D. F. Agterberg, A. Koga, and M. Sigrist, Phys. Rev. Lett. 92, 097001 (2004). 